\title{
Vibration excitation and control of a pedestrian walkway by individuals and crowds
}

\author{
James Mark William Brownjohn ${ }^{\mathrm{a}, *}$ and Tao Neng Fu ${ }^{\mathrm{b}}$

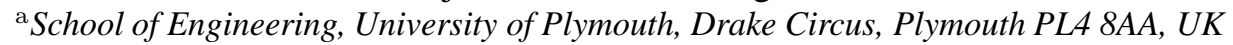 \\ ${ }^{\mathrm{b}}$ Civil Engineering and Building Department, Singapore Polytechnic, 500 Dover Road, Singapore 139651 \\ Fax: +65 67721973; E-mail: cnftao@sp.edu.sg
}

Received 24 September 2004

Revised January 2005

\begin{abstract}
As part of a continuing study on effects of humans on loading and dynamic response of footbridges, a steel frame walkway has been the subject of studies on the effects of multiple pedestrians with respect to loading and response mitigation. Following finite element modeling and experimental modal analysis to identify the low frequency vibration modes likely to be excited by normal walking, the variation of response with pedestrian density and of system damping and natural frequency with occupancy by stationary pedestrians were both studied. The potentially mitigating effect of stationary occupants is still not well understood and the study included direct measurement of damping forces and absorbed energy using a force plate. The various tests showed that energy dissipation measured directly was consistent with the observed change in damping, that vertical and lateral response both varied approximately with square root of number of pedestrians, and that the simple model of a human as a single mass-spring-damper system may need to be refined to fit observed changes in modal parameters with a crowd of humans present. Modal parameter changes with moving pedestrians were small compared to those with stationary pedestrians indicating that within limits, modal parameters for the empty structure could be used in analysis.
\end{abstract}

\section{Introduction}

Structural design normally focuses on ultimate limit states, with passing attention to serviceability through limiting deflections. Except in the case of facilities such as wafer fabrication plants where vibration control is the prime concern, vibration serviceability is often overlooked or mishandled. In recent years a number of high profile structures, such as London Millennium Bridge and Cardiff Millennium Stadium, perfectly capable of sustaining the static load of a large crowd, have 'failed' without collapse: they were found to be unable to sustain the dynamic loads of large numbers of pedestrians without inducing significant discomfort, and retrofit or restrictions on use have been required.

Footbridges are frequently lively structures and serviceability requirements such as in the British bridge design code BD37/01 [1] have provided reasonably well for limiting unacceptable response, but a number of recent developments have shown a need to revise serviceability requirements. Specifically, the phenomenon of synchronous lateral excitation (SLE) is not confined to the Millennium Bridge [2]; SLE has been observed in at least two other new structures, the Changi Mezzanine Bridge in Singapore [3] and apparently Pont Solferino, and the only mitigation possible is crowd control or enhanced damping. For vertical response, the code requirement of a maximum allowable acceleration induced by a single perfect pedestrian is either over-conservative in the case of a real (and imperfect)

*Corresponding author. E-mail: James.brownjohn@plymouth.ac. uk. 


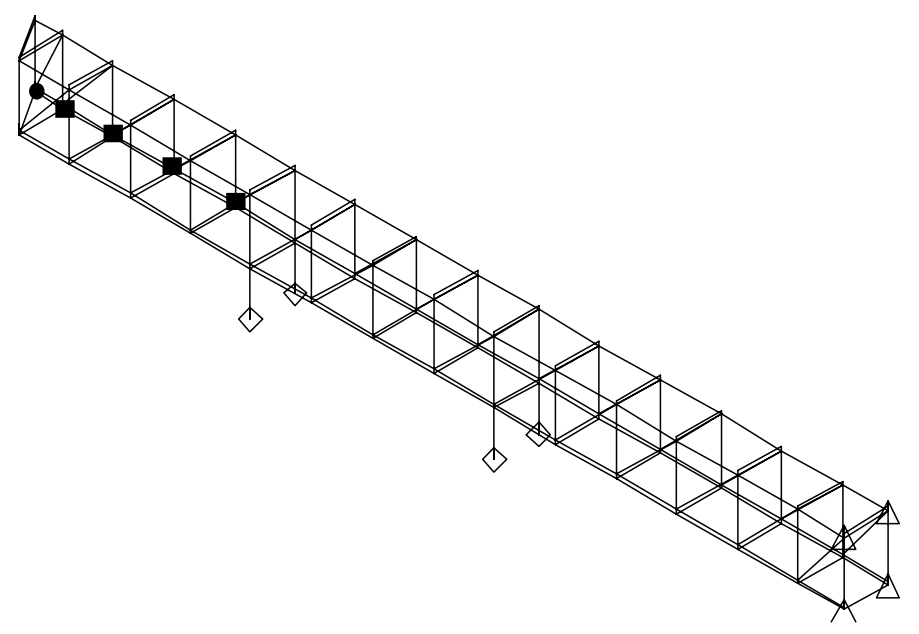

Fig. 1. Isometric View of Finite Element Model of the Bridge. Measurement points are indicated effects of stationary pedestrians, subjects stood as close as possible to point

pedestrian or inappropriate in the case of a large crowd where effects such as synchronization of movement and enhanced damping due to stationary pedestrians are not covered. For SLE the problem is now understood empirically and it is possible to predict if a problem will occur and if so, to then estimate the limiting number of pedestrians. On the other hand, the beneficial effects of human damping are not well understood and all that is known of crowd loading is that response seems to go approximately with the square root of number of pedestrians $[4,5]$.

This paper describes a comprehensive study on an unusual pedestrian bridge. It is a steel framed elevated walkway that is cantilevered at one end, hence it is relatively light, the vertical mode frequency is quite low, at $5 \mathrm{~Hz}$, and damping is also quite low. It is heavily used by students between classes, frequently empty, accessible and appears to be straightforward to model analytically.

Finite element modeling and vibration testing aimed at identifying the modal parameters is first described. Modal mass is particularly important for study of vibration serviceability yet it is surprisingly difficult to measure or predict with a degree of accuracy. As well as estimating from a finite element model and from the modal constant derived by hammer testing, a force plate was used to measure directly contact force from a jumping student which, by fitting to a model with known frequency and damping, provided probably the most reliable estimate.

In the spirit of BD37/01, response to a single perfect pedestrian was then computed and compared to response for a range of pedestrian densities measured during a period of heavy usage to add to the body of evidence on the limitations of the approach for multiple pedestrians.

Finally, the ability of stationary humans to dissipate energy was measured directly using a force plate and the capability of up to 21 stationary pedestrians (students) to increase damping capacity (to as much as 10\%) was demonstrated, a result of particular value to researchers of grandstand dynamics. By comparison, it was found that the changes in modal parameters detected for a similar number of moving pedestrians were smaller and comparable with the level of accuracy of the parameter identification.

\section{Pedestrian bridge at singapore polytechnic}

The $46 \mathrm{~m}$ long steel pedestrian bridge linking teaching Block T15 and Engineering Block T12A in Singapore Polytechnic was constructed in 1999. The bridge is a steel space truss and frame system while the deck floor comprises timber planks with a layer of stone tiling. The end at Block T15 is not structurally connected to the block except by a narrow elastomeric expansion joint, whereas at the other end, top chords and bottom chords are all bolted to the RC wall of Engineering Block T12A. 


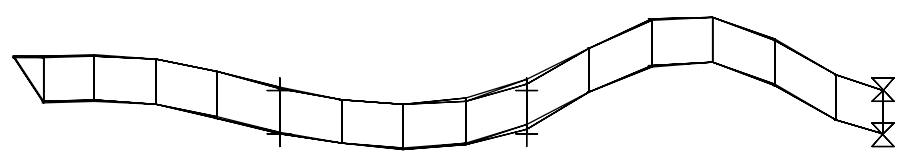

$\mathrm{Na}$ tura 1 F requency: $0.87 \mathrm{~Hz}$

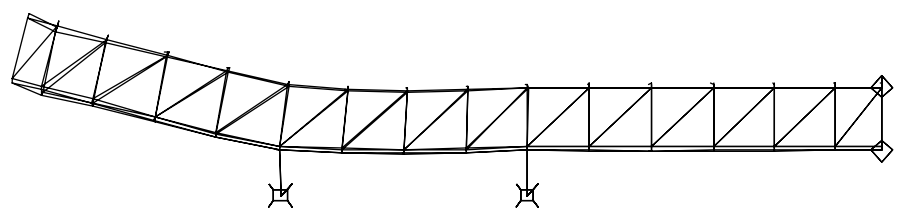

$\mathrm{N}$ a tu ra 1 F requency: $4.72 \mathrm{~Hz}$

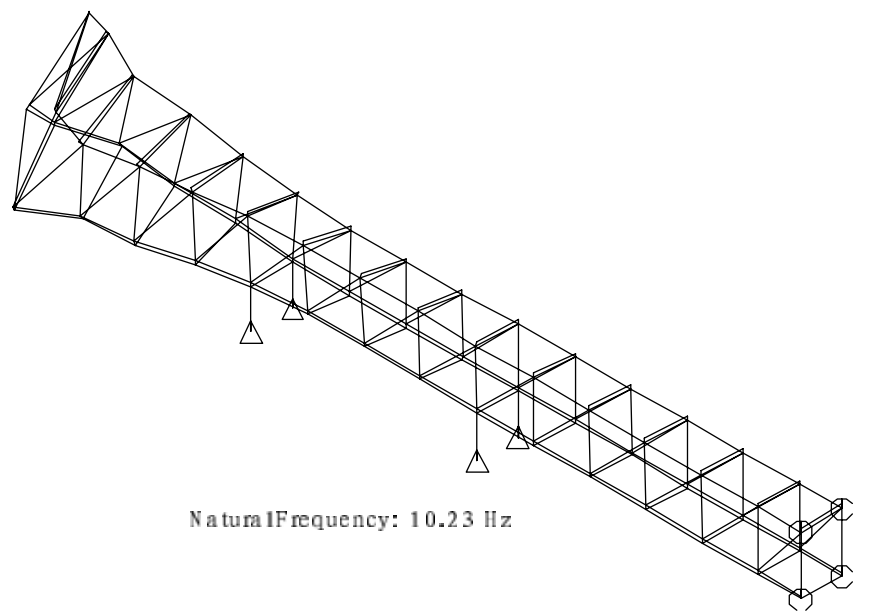

Fig. 2. Fundamental mode shapes (top to bottom) L1, V1 and T1 obtained from finite element analysis.

\section{Finite element model}

Mode shapes, natural frequencies and modal masses of the bridge were estimated from dynamic analysis using GT-STRUDL analysis software.

The bridge was modeled by incorporating all structural details according to the design concept. The four steel stanchions providing a clearance of $6 \mathrm{~m}$ above ground are assumed rigidly connected to the foundation while the top chords and bottom chords of the bridge are assumed to be pinned to the wall of Block T12A. Within the bridge, member connections are taken as rigid or pinned based on its original design assumptions. Figure 1 shows the isometric view of finite element model of the bridge.

The frequencies for 1st lateral, vertical and torsional vibration modes were estimated to be:

L1: $0.87 \mathrm{~Hz}, \mathrm{~V} 1: 4.72 \mathrm{~Hz} \mathrm{T1}: 10.2 \mathrm{~Hz}$

The corresponding mode shapes are displayed in Fig. 2.

Analysis of vertical ground reaction forces during walking [6] shows that vertical forces are concentrated at the fundamental frequency of the pacing with diminishing components at harmonics or multiples of this frequency. Lateral forces during walking are harder to measure directly, but it is clear that due to the oscillating forces required to keep balance during a cycle of two footsteps, lateral forces are concentrated at half the pacing rate, then at 1.5 and possibly 2.5 times pacing rate. Hence based on the FEM analysis it may be expected that $\mathrm{L} 1$ (at $0.87 \mathrm{~Hz}$ ) could be excited by normal walking at $1.74 \mathrm{~Hz}$ and $\mathrm{V} 1$ by the second harmonic of fast walking at $2.36 \mathrm{~Hz}$ or third harmonic of slow walking at $1.57 \mathrm{~Hz}$. Predictions of response in either mode require an accurate estimate of modal mass, 

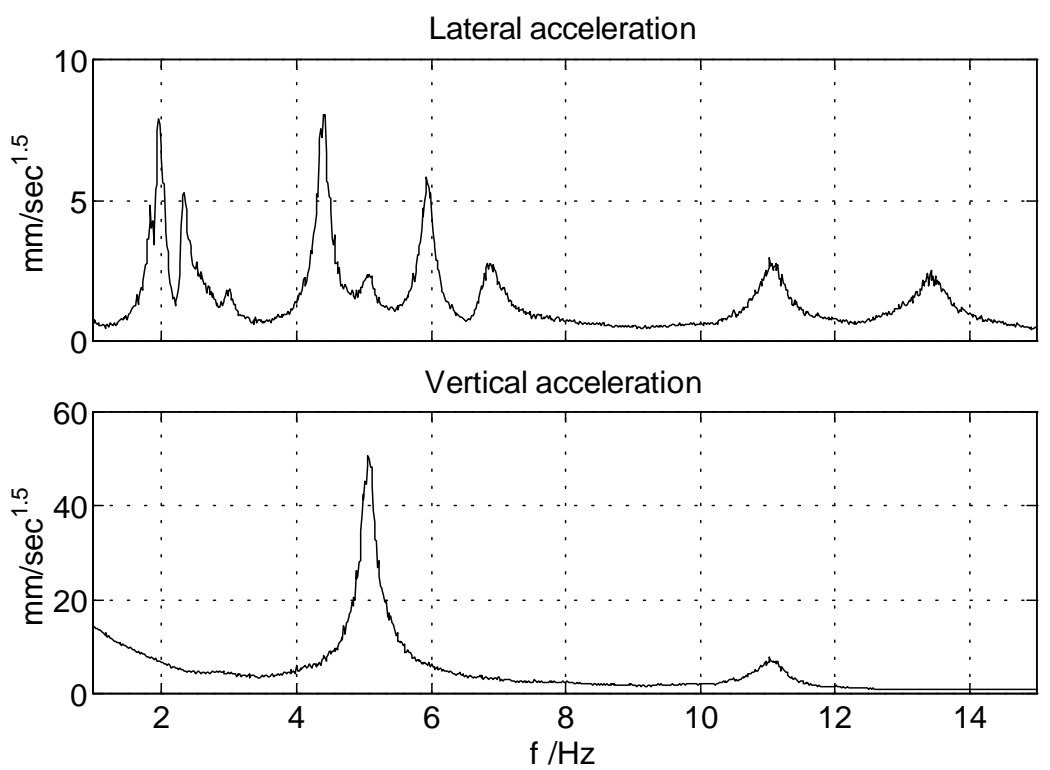

Fig. 3. Square root auto-spectral density of lateral (upper) and vertical (lower) acceleration response mainly due to heavy pedestrian activity.

which for the purposes of these studies would use unit normalization of mode shape at the free end with maximum response. This means that the ratio of response to excitation at the free end fixes a value for modal mass; for mode V1, modal mass was estimated from the FEM to be $8500 \mathrm{~kg}$, about $14 \%$ of total mass of the $60,000 \mathrm{~kg}$ bridge.

\section{Modal testing}

Figure 3 shows the square root of averaged auto-spectral density (ASD) of lateral and vertical response at the free end of the bridge to crowd loading on a busy day. Strong vertical response is seen around $5 \mathrm{~Hz}$ with strong lateral response just under $2 \mathrm{~Hz}$ and also around $4.5 \mathrm{~Hz}$. Since the range of typical walking footfall frequencies has a mean of approximately $2 \mathrm{~Hz}$ [7], relatively strong non-resonant dynamic response might be expected in the lateral direction around $1 \mathrm{~Hz}$ and in the vertical direction around $2 \mathrm{~Hz}$. The observed peaks do not fit these values hence they are likely to represent vibration modes excited by wind or by higher harmonics of the footfall forces.

To check the identity of these modes and obtain reliable modal parameter estimates, a forced vibration test was carried out using an instrumented hammer to excite vertical or lateral vibrations at the free end; Fig. 4 shows estimates of mode shape and frequency for V1 and T1 from combination of the measurements. Measurement points are indicated in Fig. 1.

Single degree of freedom circle fit [8] was used to extract modal parameters from the frequency response functions (FRFs). For vertical response, due to sensor overload, best quality FRFs were obtained for excitation at the free end and response at the midspan of the cantilever rather than at driving point hence modal mass values are not available directly corresponding to the free end of the bridge:

Mode V1: $f=4.99 \mathrm{~Hz} \quad \zeta=0.85 \%$

Mode T1: $f=11.1 \mathrm{~Hz} \quad \zeta=1.45 \%$

For lateral response FRFs were better quality but only three modes could be identified from circle fit of driving point FRF, and other modes are estimated from the ambient vibration response shown in Fig. 3. The estimated lateral modes are summarized in Table 1 . Mode L1 is believed to be the fundamental lateral vibration mode.

The FEM predictions for V1 and T1 frequencies, at $4.72 \mathrm{~Hz}$ and $10.23 \mathrm{~Hz}$ are quite close to the measured values at $4.99 \mathrm{~Hz}$ and $11.12 \mathrm{~Hz}$ respectively while the measured L1 frequency at $1.96 \mathrm{~Hz}$ is much higher than the predicted value of $0.87 \mathrm{~Hz}$, which would be due mainly to ignoring lateral stiffness from bridge wooden deck and steel roof systems. Model updating to improve predictions of lateral response was not necessary, as agreement of experiment 
Table 1

Lateral modes estimated from ambient and forced vibration testing

$\begin{array}{ccc}\text { Mode } & \text { Frequency } f / \mathrm{Hz} & \text { Damping } \zeta / \% \\ \text { L1 } & 1.962 & 1.96 \\ \text { L2 } & 2.35 & 3.7 \\ \text { L3 } & 4.414 & 1.08 \\ \text { L4 } & 6.06 & 1.16 \\ \text { L5 } & 6.88 & 3.23 \\ \text { L6 } & 13.62 & 1.79\end{array}$

mode: $1 \mathrm{f}=5.033 \mathrm{~Hz}$

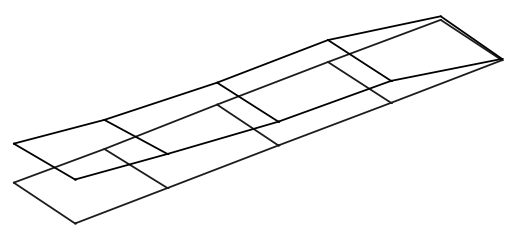

mode: $2 \mathrm{f}=11.12 \mathrm{~Hz}$

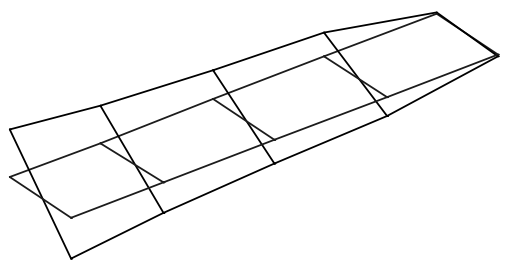

Fig. 4. Estimates of frequency and mode shape of two modes recovered from hammer testing in vertical direction, corresponding to V1 and T1.

and prediction for mode V1 was adequate. Since, judging from Fig. 3, mode V1 is the most important mode for consideration of effects of crowd loading, it was studied in detail.

\section{Detailed identification of mode V1 with human factors}

The aim of the investigation was to study all three components of the generic relationship $X=H F$ where $X$ and $F$ represent the response and loading (in time, frequency or other domains) and $H$ represents the structural system. For the study of vertical response of the bridge in mode $n$ to vertical pedestrian forces, $H$ can be represented by a single degree of freedom system with mass $m_{n}$, viscous damping coefficient $c_{n}$ and stiffness $k_{n}$ so that, for example, in Laplace domain [8]

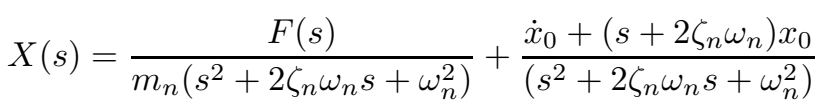

where $\zeta_{n}=c_{n} / 2 \sqrt{ } k_{n} m_{n}, \omega_{n}=\sqrt{ } k_{n} / m_{n}$ are damping ratio and natural frequency (in radians per second) of the oscillator and $\dot{x}_{0}, x_{0}$ are initial velocity and displacement.

Solution of Eq. (1) requires accurate values of $m_{n}, \zeta_{n}, \omega_{n}$. Although the frequency and damping estimates from the FRF circle fit were repeatable for the low response levels, the most reliable estimates of frequency and damping are generally obtained from the single-mode free vibration decay, which can also track non-linearity through amplitude dependence of these parameters. 


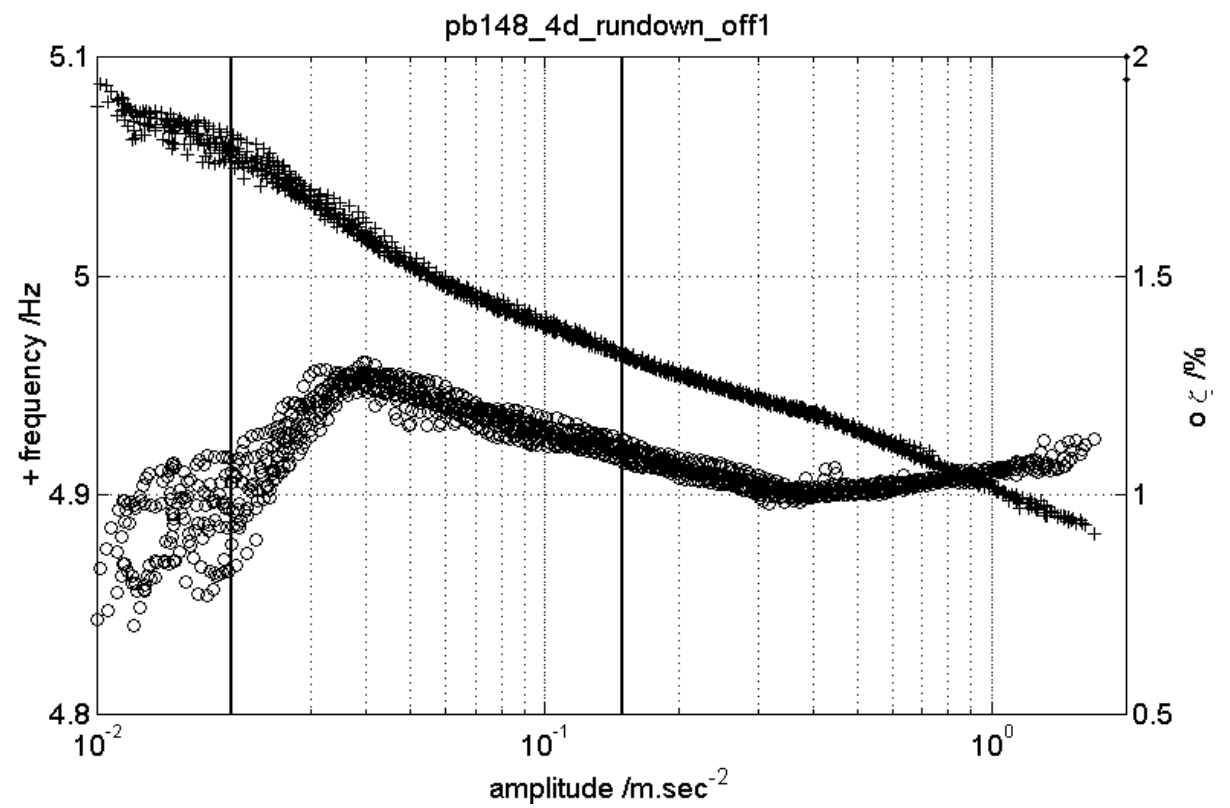

Fig. 5. Frequency $(+)$ and damping (o) estimates from free decay of mode V1 without human occupation. Solid vertical lines indicate bounds on normal pedestrian-induced response.

The solution of Eq. (1) for initial velocity and no forcing is a decaying sinusoid:

$$
\ddot{y}(t)=\ddot{y}_{\max } \sin \left(\omega_{n} t \sqrt{ } 1-\zeta_{n}^{2}\right) e^{-\omega_{n} \zeta_{n} t}
$$

and it is possible to estimate $\zeta_{n}, \omega_{n}$ from curve fitting to all or part of the free decay. Usually damping and frequency vary with amplitude so piecewise fitting using a moving window of, say eight cycles, is used to estimate parameters throughout the decay.

\subsection{Frequency and damping ratio without human occupant}

Based on the method of curve fitting to Eq. (2), Fig. 5 shows variation of mode V1 damping and frequency with amplitude merged from analysis of ten recordings of free vibration in V1. For each recording the bridge was excited by a sequence of six jumps at the free end of the bridge, indicated in Fig. 1, timed by a metronome running at 148 beats per minute (BPM), $2.47 \mathrm{~Hz}$. On the last jump the jumper jumped off the bridge (onto the adjacent landing).

As well as showing repeatability of the estimates and an inverse relationship of frequency with amplitude, the tripartite relationship of damping with amplitude and the peak at $1.3 \%$ is remarkable. The behaviour of the expansion joint must be in part responsible for the strange damping characteristic; for small oscillations it is hardly mobilized, the character is friction-like in the mid range, and at higher amplitudes of $+/-2 \mathrm{~mm}$, corresponding to $2 \mathrm{~m} / \mathrm{sec}^{2}$, the bridge is moving so much that construction joints are beginning to creak. Response of V1 to normal pedestrian traffic has amplitudes in the range 0.02 to $0.15 \mathrm{~m} / \mathrm{sec}^{2}$, (indicated in the figure by solid vertical lines) where damping averages at $1.2 \%$ and frequency around $5.0 \mathrm{~Hz}$.

\subsection{Frequency and damping ratio with human occupant}

The same exercise used to generate Fig. 5 was repeated to obtain parameter estimates with the same single $87 \mathrm{~kg}$ jumper remaining on the bridge and standing perfectly still on the bridge after the jumping sequence. The results in Fig. 6 show several features compared to Fig. 5, whose piecewise best-fit trend-lines are given in Fig. 6 for comparison.

As well as a drop in frequency of approximately $0.02 \mathrm{~Hz}$ at all amplitudes, damping has increased, by approximately $0.4 \%$ at amplitudes greater than $0.04 \mathrm{~m} / \mathrm{sec}^{2}$, although the values show considerable scatter. 


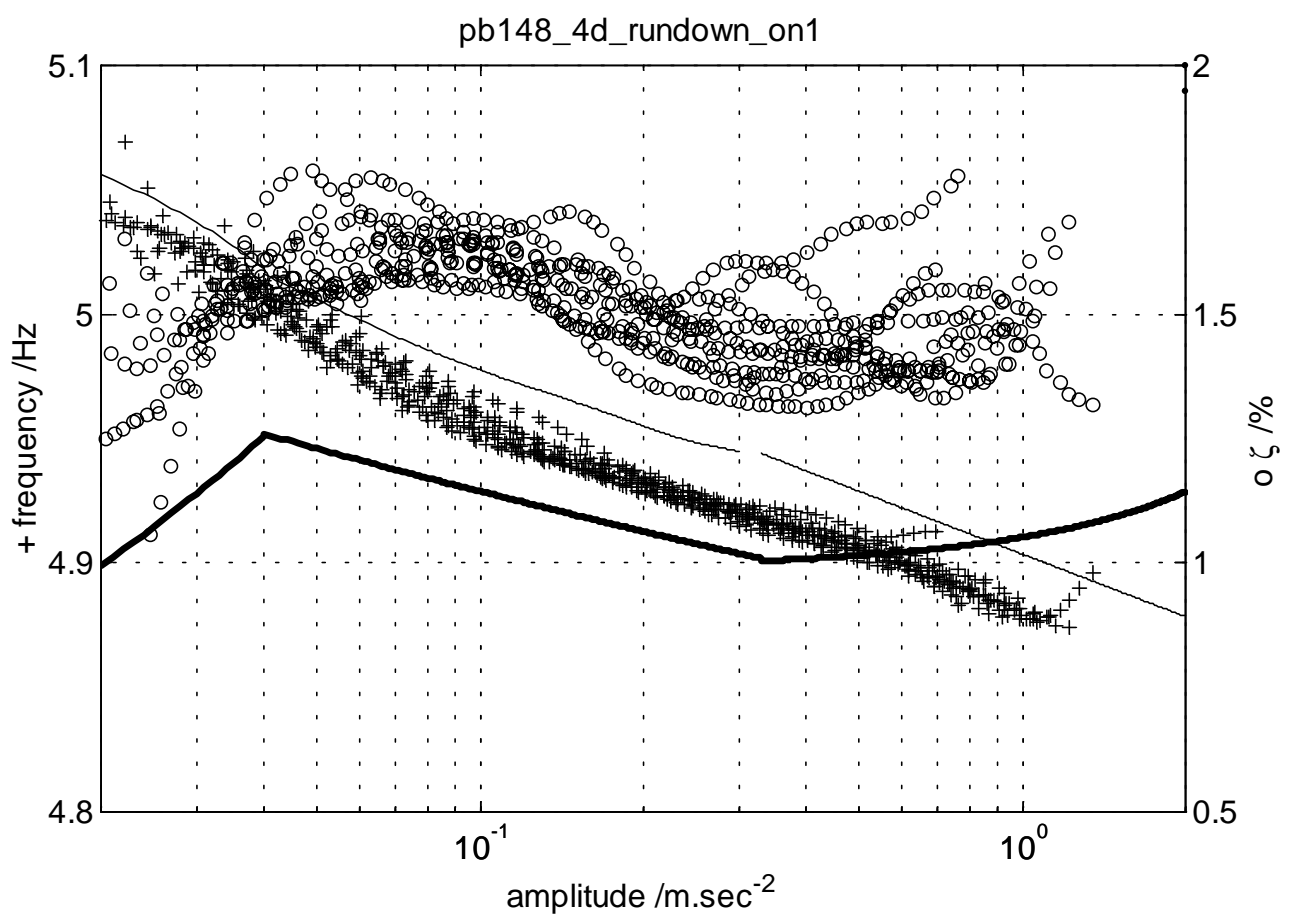

Fig. 6. Frequency (+) and damping (o) estimates from free decay of mode V1 with single human occupant. Values for empty bridge (from Fig. 5) are shown as solid lines.

\subsection{Modal mass}

As the estimates of modal mass via hammer testing were not reliable and disagreed with FEM predictions, a force plate was used to estimate mass via measurement of force together with response at a single point due to a human jumping. The top plot of Fig. 7 shows measured acceleration response due to an $80 \mathrm{~kg}$ male jumping at the free end of the bridge to build up a resonant response through the second harmonic of the jumping frequency. The middle plot shows the input force recorded using a single axis force plate. The data up to 62 seconds were used to estimate modal mass as follows.

The measured force signal was used as input to a simulation using Eq. (1) for $n=\mathrm{V} 1$ with mass $m=1000 \mathrm{~kg}$, $\omega=2 \pi \times 5 \mathrm{~Hz}$ and $\zeta=1.3 \%$. The bottom plot of Fig. 7 shows the result. For the initial build up, before the effect of damping becomes significant, the increment of acceleration is inversely proportional to mass, hence the modal mass can be estimated by comparing acceleration increment per cycle between measurement (top plot, $0.08 \mathrm{~m} / \mathrm{sec}^{2}$ ) and simulation (bottom plot, $0.59 \mathrm{~m} / \mathrm{sec}^{2}$ ), leading to an estimate of modal mass $m_{V 1}=7,380 \mathrm{~kg}$.

\subsection{Mode VI energy input and extraction due to single occupant}

The data of Fig. 7 after 62 seconds reveal the effect of the occupant on damping and frequency of the occupied bridge. The same process of time domain curve-fitting to free decay that was used to obtain the data points in Fig. 6 was used on last few seconds of data shown in Fig.7 to demonstrate that the presence of this test subject at the free end of the bridge increases damping by approximately $0.3 \%$. It is certain that none of the extra damping is due to the force plate itself.

Close examination of the top two plots of Fig.7 shows that during the free decay part of this jumping sequence (and other similar measurements) when the jumper is stationary, the phase angle between the component of force at the bridge frequency switches from leading response by $90^{\circ}$ to lagging response by approximately $155^{\circ}$.

The observed differences with and without a human occupant are consistent with the accepted notion that a stationary human acts as a passive damper. While jumping, the effect is as an external force, and the direct 

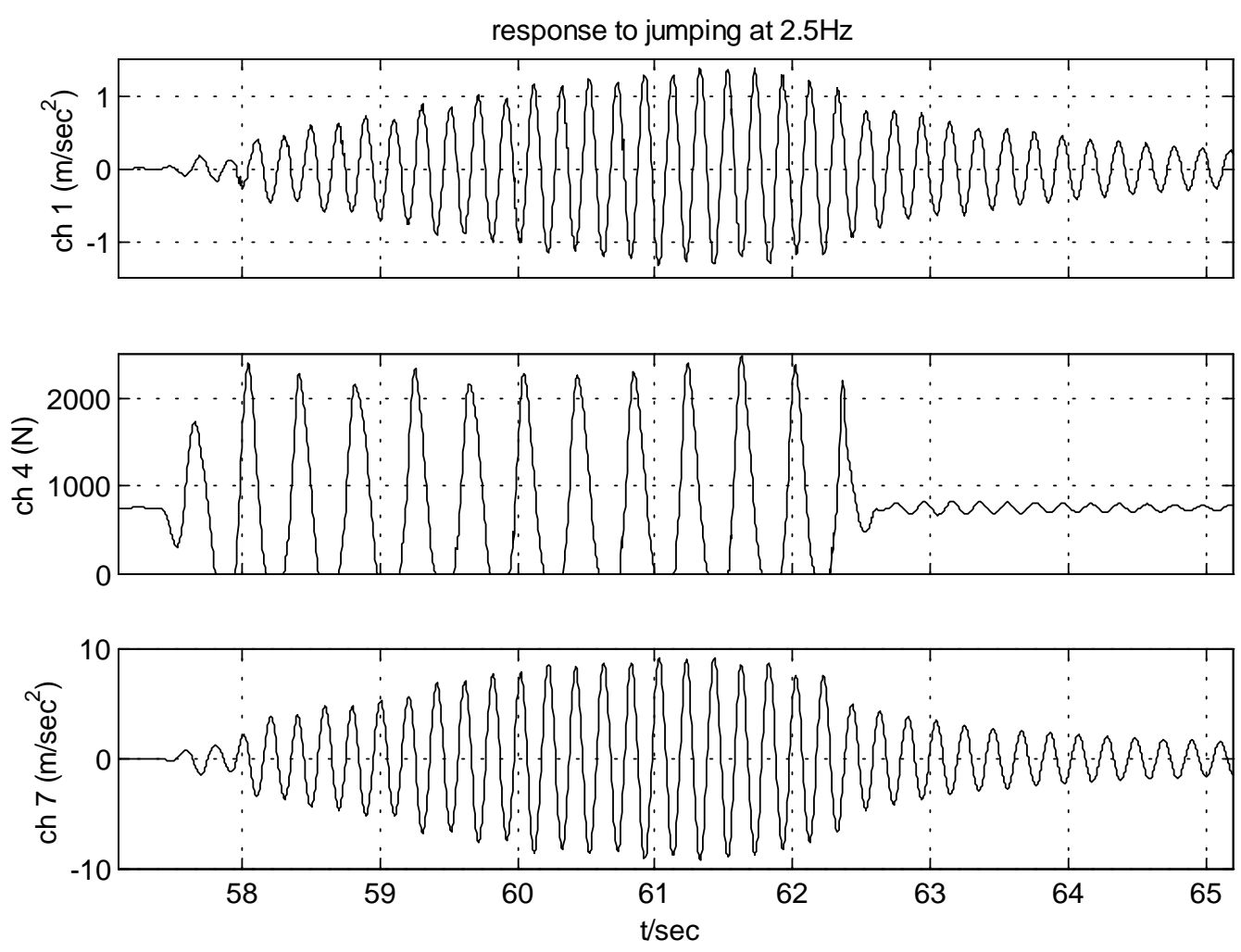

Fig. 7. $80 \mathrm{~kg}$ test subject jumping at $2.5 \mathrm{~Hz}$. top: measured acceleration response middle: measured contact force Bottom: simulated acceleration response for $1000 \mathrm{~kg}$ mass.

measurement of contact force isolates any influence of jumper dynamics, hence the modal mass estimate should be reliable. In the decay phase, since the contact force has been recorded, the mechanism of the enhanced damping could be studied. In fact it should be possible to estimate energy input (before 62 seconds) and energy extraction (after 62 seconds).

Figures 8 and 9 show the power and its time integral (work done or energy) by the jumper during the forcing and decay phases of Fig. 7, i.e. before and after 62 seconds. The power is obtained from the product $F$ jumper $\times v_{\text {plate }}$ where $v_{\text {plate }}$ is the plate velocity derived by integration of acceleration data shown in the top plot of Fig. 7 . In the forcing phase the power averages 5 Joules/sec (Watts) and when the response reaches a maximum or steady state it could be argued that 5 Watts must also equal the energy dissipation of the bridge.

With this line of argument, since the $5 \mathrm{~W}$ rate of energy dissipation (power) due to bridge damping in mode $\mathrm{V} 1$ is related to damping ratio by $2 \zeta V_{1} \omega_{V 1} \times m_{V 1} \times v_{\text {plate }}^{2}$, an estimate of mode V1 structural damping $\zeta_{V 1} \approx 1.3 \%$ can be obtained given values of $m_{V 1}, \omega_{V 1}, \sigma^{2}\left(v_{\text {plate }}\right)$ where $\sigma^{2}$ represents variance.

The bottom plot of Fig. 9 shows the kinetic energy of the bridge as the vibration decays; the maxima of each cycle represent the total of potential and kinetic which is slowly reduced by internal and external damping losses during the decay cycles and the highest maximum is 1.8 Joules. Of the 1.8 Joules that has been dissipated, the middle plot shows that 0.3 Joules is work done or energy dissipated due to the stationary human. The human contribution of 0.3 Joules to a total energy loss of 1.8 Joules matches quite well the observed $0.3 \%$ increase in damping to $1.6 \%$ between Figs 5 and 6.

\subsection{Mode V1 pedestrian-induced vibration control by stationary humans}

The ability of a stationary human to dissipate energy has been demonstrated clearly and it should be worthwhile to investigate the effect of different stationary humans in mitigating vibrations induced by pedestrians. Because 

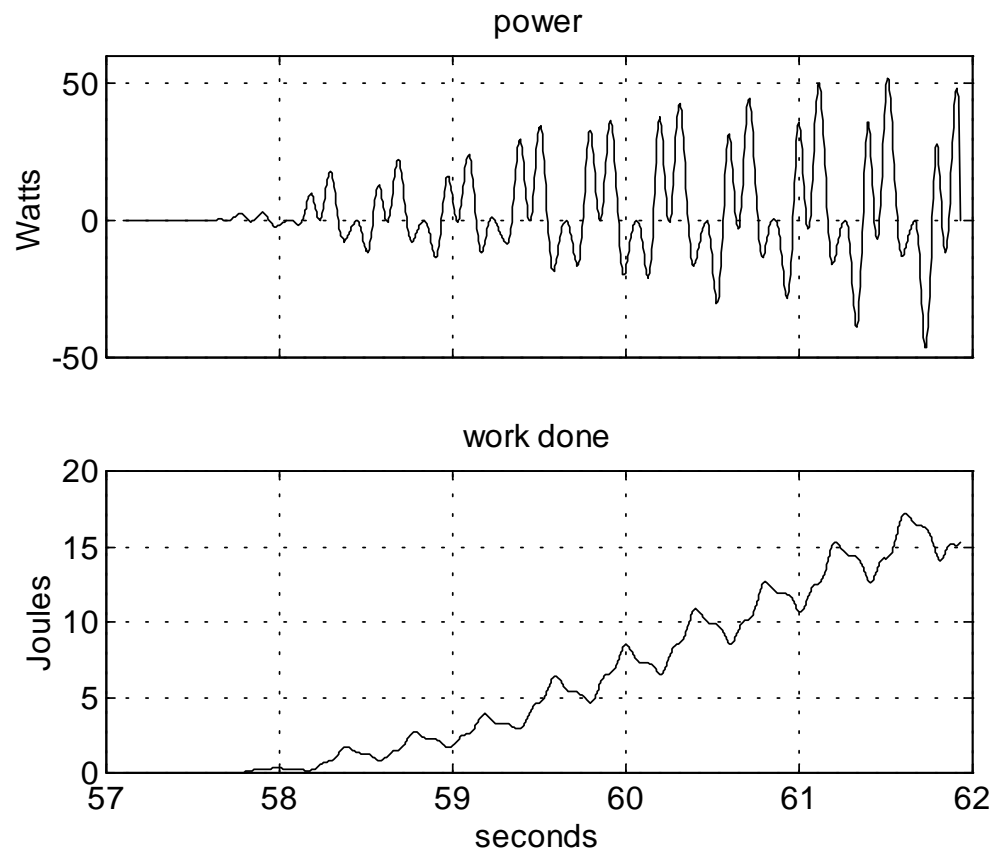

Fig. 8. Upper: Power supplied to bridge derived from contact force and acceleration. Lower: Time integral of power; energy supplied to bridge.

different subjects (human dampers) were used and because a perfectly repeatable loading could not be generated, the additional damping was studied from the ratio of measured energy loss in a cycle to total energy.

The effective viscous damping is defined via the ratio of energy loss per cycle of oscillation, $\Delta E$, to total stored energy $E$ i.e. [9].

$$
\zeta=\Delta E / 4 \pi E \text {. }
$$

Work done (hence energy loss) by a human damper on a force plate during one cycle of harmonic oscillation lasting $T$ seconds is given by

$$
\Delta E=\int_{0}^{T} F_{\text {plate }} v_{\text {plate }} d t=(T / 2) \widehat{F}_{\text {plate }}\left(\widehat{a}_{\text {plate }} / \omega\right) \sin \phi
$$

where $\widehat{F}_{\text {plate }}, \widehat{a}_{\text {plate }}$ are amplitudes of force and acceleration, $\phi$ is phase angle between acceleration and force measured on the force plate, and total energy $E$ is peak kinetic energy defined as

$$
E=(1 / 2) m_{V 1}(\widehat{a} / \omega)^{2} .
$$

Since cycle duration $T=2 \pi / \omega$, the additional human contribution to damping ratio is given by

$$
\zeta_{h \text { add }}=\frac{(\pi / \omega) \widehat{F}_{\text {plate }}\left(\widehat{a}_{\text {plate }} / \omega\right) \sin \phi}{4 \pi(1 / 2) m_{V 1}\left(\widehat{a}_{\text {plate }} / \omega\right)^{2}}=\begin{aligned}
& \widehat{F}_{\text {plate }} \sin \phi \\
& 2 m_{V 1} \widehat{a}_{\text {plate }}
\end{aligned} .
$$

A number of individuals were asked to stand on a force plate (located at the free end of the bridge) while recording the response to a pedestrian walking normally, as shown in Fig. 10. Due to the normalization with respect to kinetic energy, the actual values of response and force are less important than having a steady state for long enough to identify the force, acceleration and phase angles. From the walking experiment, taking $m_{V 1}=7,380 \mathrm{~kg}$, values of $\widehat{F}_{\text {plate }} / \widehat{a}_{\text {plate }}, \phi$ and body mass $m_{h}$ for seven individuals are given in Table 2 . There is considerable variation of damping potential among individuals but the coefficient of variation (COV) shows this is reduced with normalization with respect to body mass. 
Table 2

Damping and energy dissipation parameters for seven students

$\begin{array}{ccclcc}\text { Student } & m_{h} / \mathrm{kg} & \widehat{F}_{\text {plate }} / \widehat{a}_{\text {plate }} / \mathrm{kg} & \phi & \zeta_{h \text { add }} / \% & \zeta_{h \text { add }} / m_{h} \\ \# 1 & 53 & 95.5 & -22.5 & 0.24 & 46 \mathrm{e}-6 \\ \# 2 & 56 & 104 & -21 & 0.25 & 44 \mathrm{e}-6 \\ \# 3 & 69 & 136 & -12.5 & 0.20 & 29 \mathrm{e}-6 \\ \# 4 & 42 & 86 & -15 & 0.16 & 39 \mathrm{e}-6 \\ \# 5 & 49 & 96 & -11 & 0.13 & 27 \mathrm{e}-6 \\ \# 6 & 87 & 163 & -21.5 & 0.40 & 46 \mathrm{e}-6 \\ \# 7 & 80 & 141 & -14.7 & 0.24 & 29 \mathrm{e}-6 \\ \text { COV }(\%) & & & & 37.5 & 23\end{array}$

power
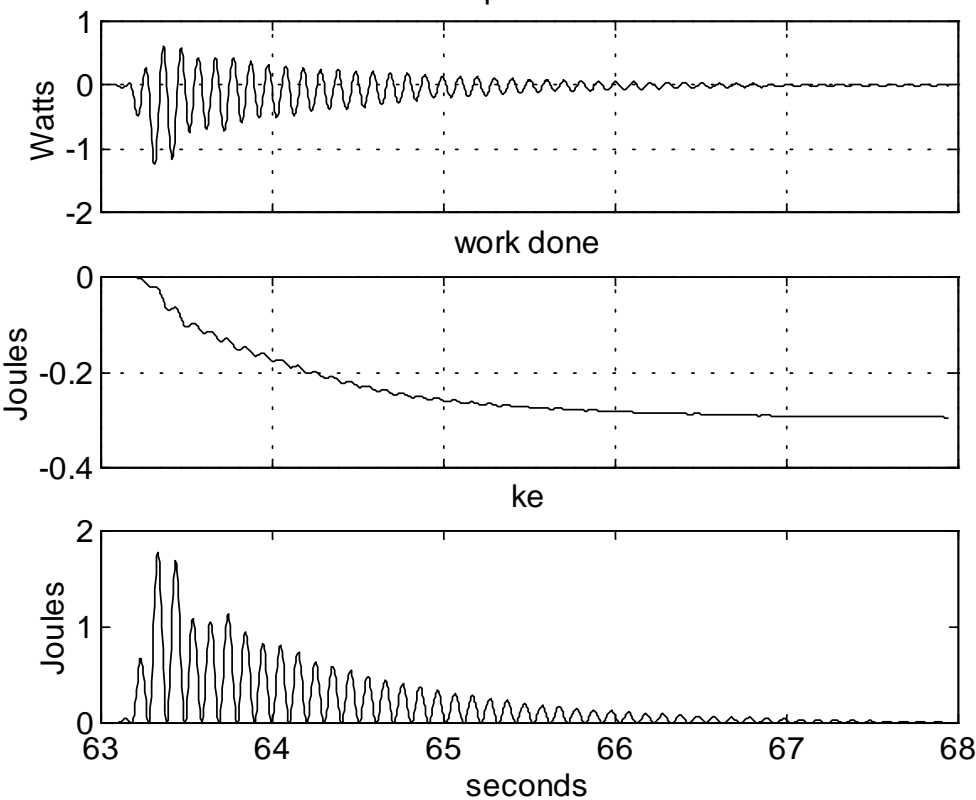

Fig. 9. Top: Energy dissipation rate (power) due to human occupant Lower: Time integral of power, accumulated energy dissipation due to occupant, 0.3 Joules taken out by the occupant Bottom: Total Kinetic energy, maximum 1.8 Joules.

\section{Crowd effects: variations of loading response, damping and frequency with multiple human occupants}

\subsection{Variation of V1 and L1 response and modal parameters with number of stationary pedestrians}

The response at the free end of the bridge in modes V1 and L1 was tracked during a 136-minute period when the bridge was heavily used during an exhibition set up close to one end of the bridge. The number of people $N$ passing the free end of the bridge during 30-second periods was tracked with a moving window shifting 3 seconds. $N$ does not exactly represent the number of people on the bridge, but it is an indication, and 30 seconds is the estimated time taken to cross the whole bridge at a leisurely pace. Figure 11 shows variation of RMS response within each 30 -second period with corresponding value of $N$. The dependence on square root of $N$ is clear, being consistent with previous observations and models of recently developed models of crowd loading for vertical response [2,6].

Vertical response is almost entirely in mode V1 whereas lateral response is spread evenly across the first four lateral modes L1 ... L4 identified in Table 1. Figure 11 thus not only provides useful calibration for the models of vertical loading but provides evidence for similar models of lateral loading yet to be developed for the usual situation where synchronization with low frequency modes is not an issue.

It has been suggested that the presence of a large number of pedestrians modifies the modal properties of the bridge, although it is generally taken that moving pedestrians act as load generators, in which case the effect cannot 


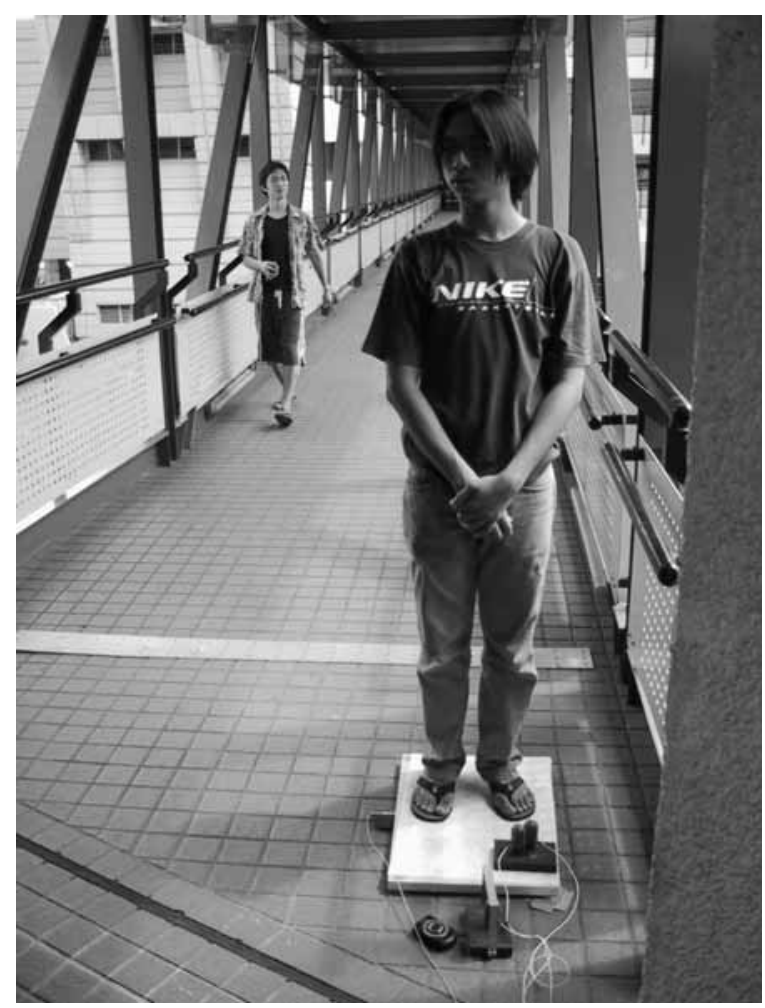

Fig. 10. Measurement of mitigating effect of stationary occupant on walking-induced response.
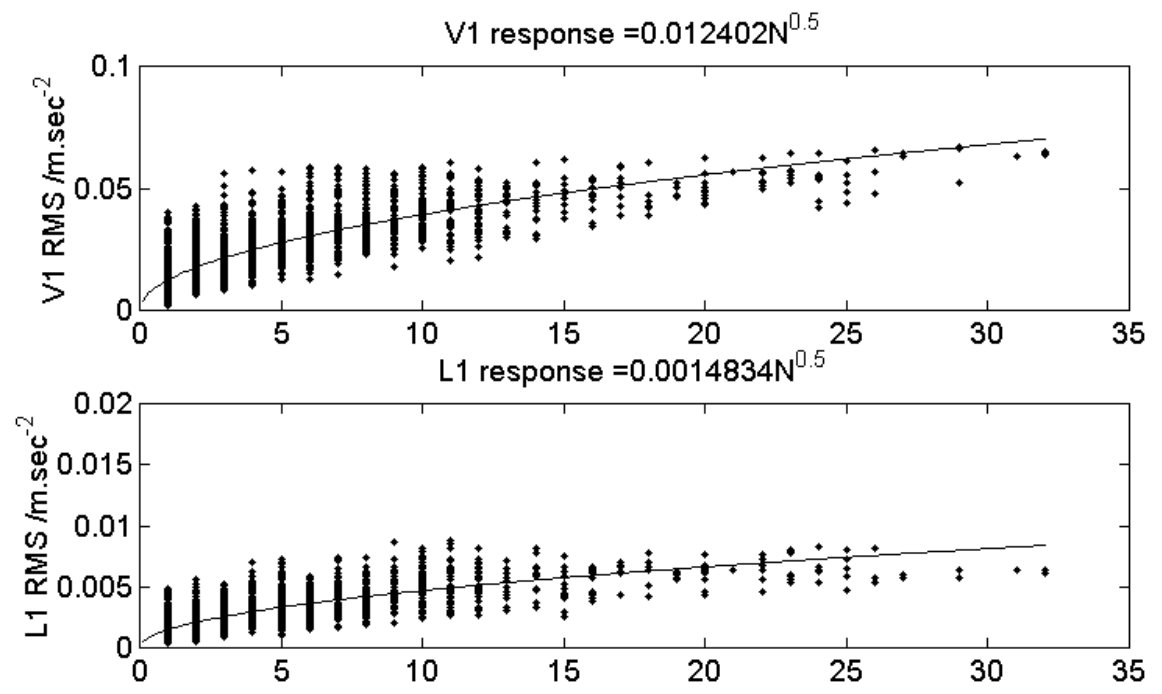

Fig. 11. Variation of RMS modal response with crowd numbers.

be modeled simply using the SDOF human models proposed for stationary humans. Figure 12 shows estimates of frequency and damping ratio corresponding to the data points of Fig. 11. The only way possible to obtain such values was from 'output-only' parameter estimation techniques, in this case by curve fit to the auto power spectra the 30-second response samples. There are unavoidable flaws in this approach, not least due to the non-stationarity 

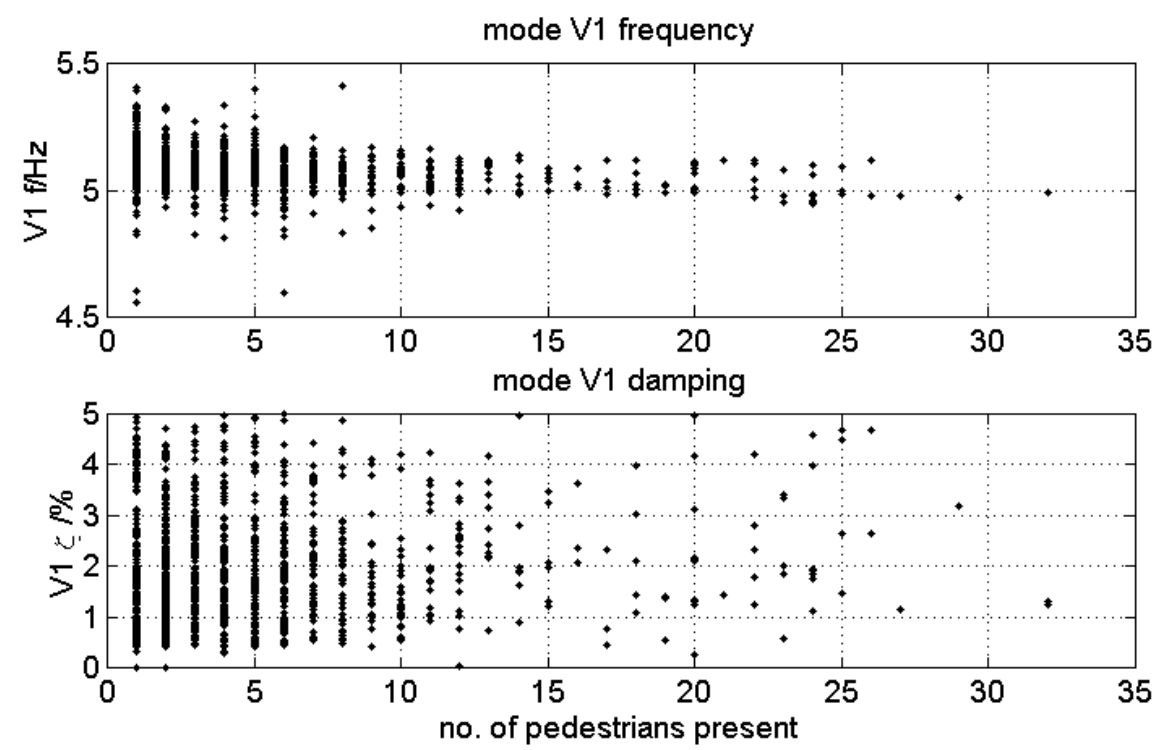

Fig. 12. Estimates of vertical mode frequency and damping with pedestrians walking across bridge.

and variable frequency content of the forcing function and even with inclusion of only those records having good quality of fit, the absolute accuracy of the values should not be relied on. Nevertheless what is clear is that mode V1 frequency values are generally consistent with values for the empty bridge at relatively low amplitude with minimal reduction due to increasing response. For damping the scatter does not allow for any trend to be detected.

More reliable observations from a test on another much larger bridge [3] indicate that lateral vibration mode parameters are barely affected even with large numbers of moving pedestrians, while for vertical response moving pedestrians enhance damping slightly, but more so when stationary.

The peak vertical response of the bridge was $+/-0.3 \mathrm{~m} / \mathrm{sec}^{2}$. Using the BD37/01 criterion, assuming a pedestrian moving at $2 \mathrm{~m} / \mathrm{sec}$ with a footfall rate of $5 \mathrm{~Hz}$ and a reduction of $70 \%$, the predicted peak response is $0.105 \mathrm{~m} / \mathrm{sec}^{2}$, a value exceeded frequently during the measurement.

\subsection{Variation of mode V1 damping and frequency with stationary pedestrians}

The final experiment involved a study of variation of natural frequency and damping ratio of the bridge as the number of human occupants increased from zero to 21 then back to zero. As occupants were added or removed, the free decay of the bridge was studied using a heel drop, having the aim to investigate the effect of occupants on a range of bridge modes. The bridge occupants, or a subset of them, provided the heel drop, and free decay response beginning with amplitude $0.15 \mathrm{~m} / \mathrm{sec}^{2}$ was (for consistency) used to extract damping and frequency estimates.

Figure 13 shows the variation of estimates, which became difficult to obtain as damping increased. The slope for additional damping is $0.26 \%$ (absolute) per person, and for reduction in frequency is $-0.26 \%$ (relative to unloaded frequency) per person. The damping and frequency values for the unoccupied bridge had changed slightly during the test.

\subsection{Modelling the human as a SDOF system}

An attempt was made to explain the variation of modal parameters observed in Fig. 13 using an established simplified model of the human dynamic system.

The approach was to model the group of humans as individual or combined SDOF systems attached to the bridge, and determine the parameters of the combined multi- or two degree of freedom system. For this purpose, values of human damping coefficient and stiffness would be needed. 
Table 3

Human parameters from literature (rows 1-3) and as identified from crowd test (rows 4-8)

\begin{tabular}{lccccc} 
Mass parameters & \multicolumn{2}{c}{ Fitted human parameters } & \multicolumn{2}{c}{ Modal parameters } \\
Human mass & $\begin{array}{c}\text { bridge modal } \\
\text { mass } m_{V 1} / \mathrm{kg}\end{array}$ & $k_{h}(\mathrm{kN} / \mathrm{m})$ & $c_{h}(\mathrm{Ns} / \mathrm{m})$ & $f_{h}(\mathrm{~Hz})$ & $\zeta_{h}(\%)$ \\
$0.9 \times 58$ (Sachse [10]) & - & 71 & 1270 & 5.87 & 33 \\
80 (Brownjohn [12]) & - & 82 & 1950 & 5.27 & 36 \\
Various (Zheng [13]) & - & - & - & $5.24 \pm 0.4$ & $39 \pm 5$ \\
$0.9 \times 58$ & $7380(\mathrm{FP})$ & 44.3 & 1790 & 4.64 & 59 \\
$0.8 \times 58$ & $7380(\mathrm{FP})$ & 45.2 & 1440 & 4.97 & 50 \\
$0.9 \times 58$ & $8500(\mathrm{FEM})$ & 52 & 1590 & 5.02 & 48 \\
$0.8 \times 58$ & $8500(\mathrm{FEM})$ & 50 & 1240 & 5.23 & 41 \\
$0.8 \times 87$ & $8500(\mathrm{FEM})$ & 76 & 1810 & 5.25 & 39
\end{tabular}

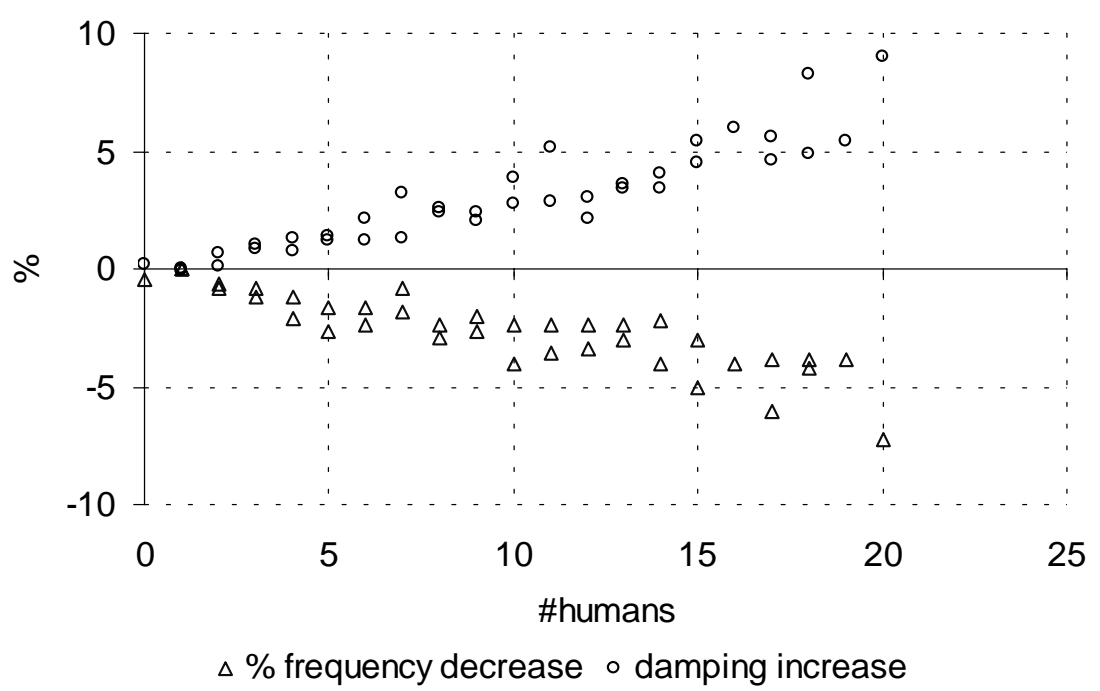

Fig. 13. Absolute changes in $\%$ damping and $\%$ changes in frequency due to human occupation.

A comprehensive study of the effect of human damping has been conducted by Sachse [10] who identified modal properties for sitting humans. Sitting and standing humans have similar effect [11], and for average mass of a student $\left(m_{h}=58 \mathrm{~kg}\right)$, Sachse's human model 'A' with effective human mass $m_{h}=\alpha m, \alpha=0.9, f_{h}=5.87$ and $\zeta_{h}=33 \%$ should have $k_{h}=71 \mathrm{kN} / \mathrm{m}$ and $c_{h}=1270 \mathrm{Ns} / \mathrm{m}$ but these last two parameters are only indicative. Other experimental data provide similar values: the human natural frequency is in the range $5-6 \mathrm{~Hz}$, damping from 30-40\%.

The original (unoccupied) mode V1 frequency and damping are taken as $5 \mathrm{~Hz}$ and $1.3 \%$, but the modal mass is assumed not to be known precisely. For unit mode shape at the tip of the free end that value is $m_{V 1}=7,380 \mathrm{~kg}$ from the force plate, (FP) a result which should be reliable, or $m_{V 1}=8,500 \mathrm{~kg}$ from the FEM, a result which may better fit the results. For the location where the students were standing the effective modal mass would be scaled up by squared inverse of mode shape $\psi \approx 0.9$, providing effective masses at the centre of the group as either $9,100 \mathrm{~kg}$ or $10,500 \mathrm{~kg}$.

For the two possible values of bridge modal mass and several different values of human mass, Newton's method was used to solve for unknown human stiffness and damping parameters $k_{h}$ and $c_{h}$ in a two degree of freedom model of the bridge and human(s) that best fitted the frequency and damping change per person of Fig. 13. The values of $\alpha<1$ were used as indicated in order to obtain agreement with both the modal parameter change per person and the linear trend of Fig. 13.

The human parameters that fit the data of Fig. 13 are given in Table 3 and compared with published data for human parameters from three different sources $[10,11,13]$. 
The last row is for the data of Figs 5 and 6 for one $87 \mathrm{~kg}$ male. Again, a reduced body mass and increased bridge mass is required for a good fit, and in this case damping is measured at the point of unit mode shape.

Compared with published data it would seem that reasonable agreement can only be obtained using larger modal mass with smaller human mass. One possible explanation for the discrepancy is due to considering the bridge as a SDOF system, which may be acceptable if added mass is small compared to modal mass. As the total human mass exceeds $1000 \mathrm{~kg}$, a distributed mass model should work better, yet the same analysis still shows human parameters that still struggle to match published values. The SDOF simplification is popular in this application and is, up to a point, capable of representing the behaviour of multi-degree of freedom model [14,15] that could be used as alternatives. In fact the difficulties in obtaining a good fit with the simple models suggest that their use be reviewed.

\section{Discussion and conclusions}

The study has provided the opportunity to address a number of contentious issues relating to vibration serviceability of footbridges.

First, the ability of a human to act as a damper, mentioned in many researches and code provisions, has been identified directly using measurements of the human force that damps vibration during free decay and transit of other pedestrians. The effect on damping matches observed changes in damping values.

Second, the variation of response with crowd numbers appears to go with the square root of number of pedestrians, as mentioned by other researchers but this exercise has demonstrated the correlation for lateral as well as vertical response. Usually the code provision based on perfect first harmonic forcing is adequate but this case shows much stronger levels can be achieved by second (or third) harmonic forcing. Definitive guidance on loading due to multiple pedestrians is still lacking in bridge codes.

Thirdly, the increase in damping and decrease in frequency with crowd size for stationary pedestrians has been demonstrated, yet it is curious that the extracted stiffness and damping parameters do not fit well to published models which represent the human system as single degree of freedom mass-spring-dashpot system. This shows that such model may be too simplistic and suggests the need for extra research for the case of large crowds.

The damping and frequency changes observed with varying numbers of moving pedestrians were indistinct but clearly far smaller than those observed due to stationary pedestrians. This supports existing understanding that response prediction models may give reasonable results if moving pedestrian pedestrians are taken purely as load generators. Also, while presence of stationary pedestrians cannot be relied on, the probable numbers of stationary pedestrians would increase with crowd numbers, particularly if movement is restricted, leading to a self-limiting mechanism for vertical vibrations with high crowd density.

\section{Acknowledgements}

The authors are grateful to the students of Singapore Polytechnic and to Pilate Moyo and Niels Macheel for their help with the experimental work on the bridge.

\section{References}

[1] The Highways Agency, BD37/01 Steel, concrete and composite bridges: Specifications for loads, Part 2, Appendix C, 2001.

[2] P. Dallard, A.J. Fitzpatrick, A. Flint, S. Le Bourva, A. Low, R.M. Ridsill Smith and M. Willford, The London Millennium Footbridge The Structural Engineer 79(22) (2001), 17-33.

[3] J.M.W. Brownjohn, P. Fok, M. Roche and P. Omenzetter, Long span steel pedestrian bridge at Singapore Changi Airport. Part 2: Crowd loading tests and vibration mitigation measures, The Structural Engineer 82(16) (2004), 28-34.

[4] J.E. Wheeler, Prediction and control of pedestrian-induced vibration in footbridges, ASCE Journal of Structural Engineering 108(9) (1982), 204-2065.

[5] Y. Matsumoto, T. Nishioka, H. Shiojiri and K. Mastsuzaki, Dynamic testing of footbridges, IABSE Proceedings, P-17/78, August, 1978.

[6] J.M.W. Brownjohn, A. Pavic and P. Omenzetter, A spectral density approach for modelling continuous vertical forces on pedestrian structures due to walking, Canadian Journal of Civil Engineering 31(1) (2004), 65-77. 
[7] H. Bachmann and W. Ammann, Vibrations in structures induced by man and machine, IABSE Structural Engineering Documents 3E, 1987. ISBN 3-85748-052-X.

[8] N.M.M. Maia, J.M.M. Silva, J. He, N.A.J. Lieven, R.M. Lin, G.W. Skingle, W. To and A.P.V. Urgueira, Theoretical and Experimental Modal Analysis, Research Studies Press Ltd, 1997.

[9] J.M.W. Brownjohn, 'Estimation of damping in suspension bridges'. Structures and Buildings, Proceedings, Institution of Civil Engineers 104 (1994), 401-415.

[10] R. Sachse, The influence of human occupants on the dynamic properties of slender structures, PhD thesis, University of Sheffield, April, 2002, Dr Andere Verlag, Osnabruck.

[11] J.M.W. Brownjohn and X. Zheng, The effects of human posture on energy dissipation from vibrating floors, Proceedings of SPIE 4317 (2001), 489-493.

[12] J.M.W. Brownjohn, Energy dissipation from vibrating floor slabs due to human-structure Interaction, Shock and Vibration 8(6) (2001), $315-323$.

[13] X. Zheng and J.M.W. Brownjohn, Modeling and simulation of human-floor system under vertical vibration, Proceedings of SPIE 4327 (2001), 513-520

[14] M.J. Griffin, Handbook of human vibration, Academic Press, London, 1990.

[15] International Standards Organisation, Mechanical vibration and shock - Mechanical transmissibility of the human body in the z direction, ISO 7962, 1987. 

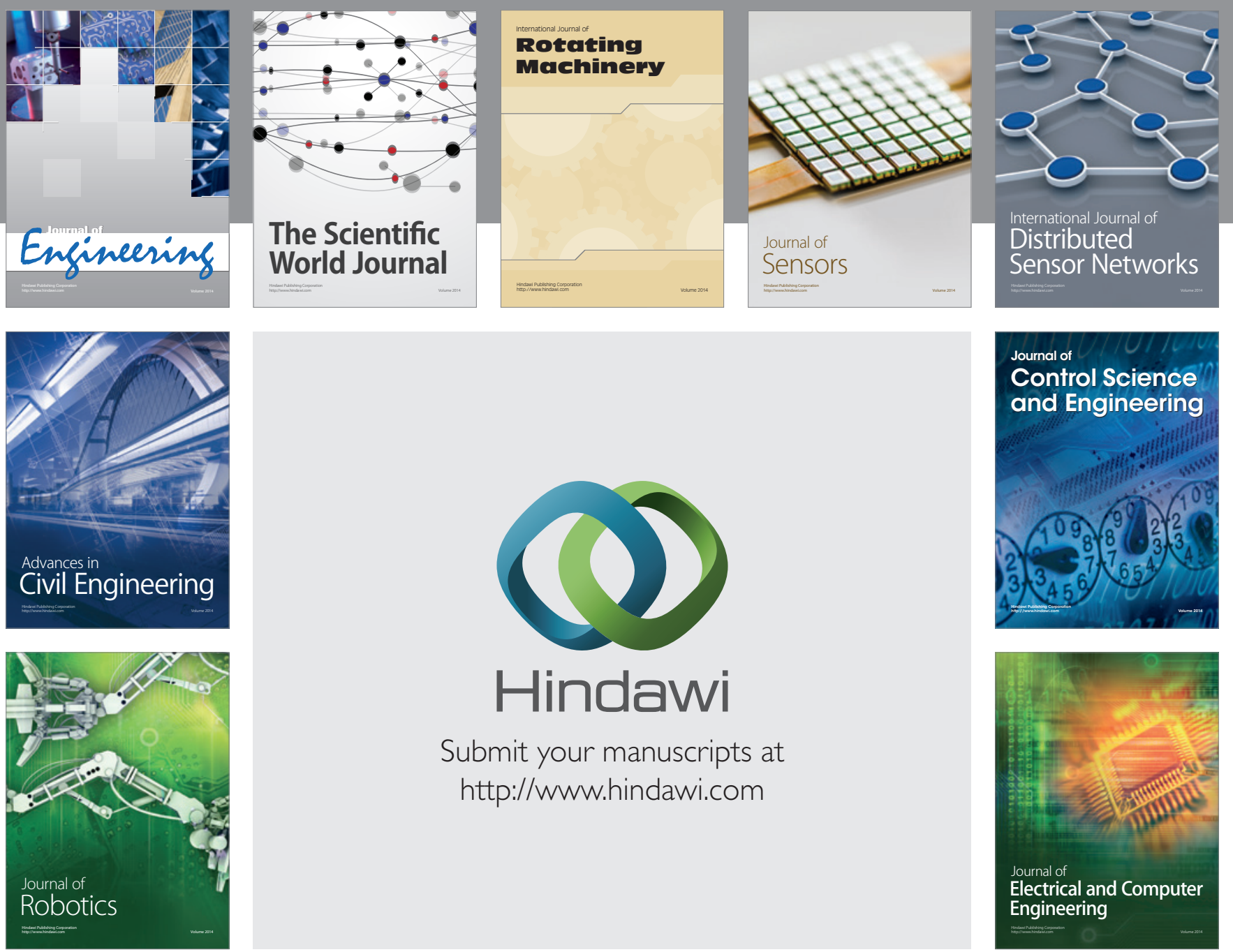

Submit your manuscripts at

http://www.hindawi.com
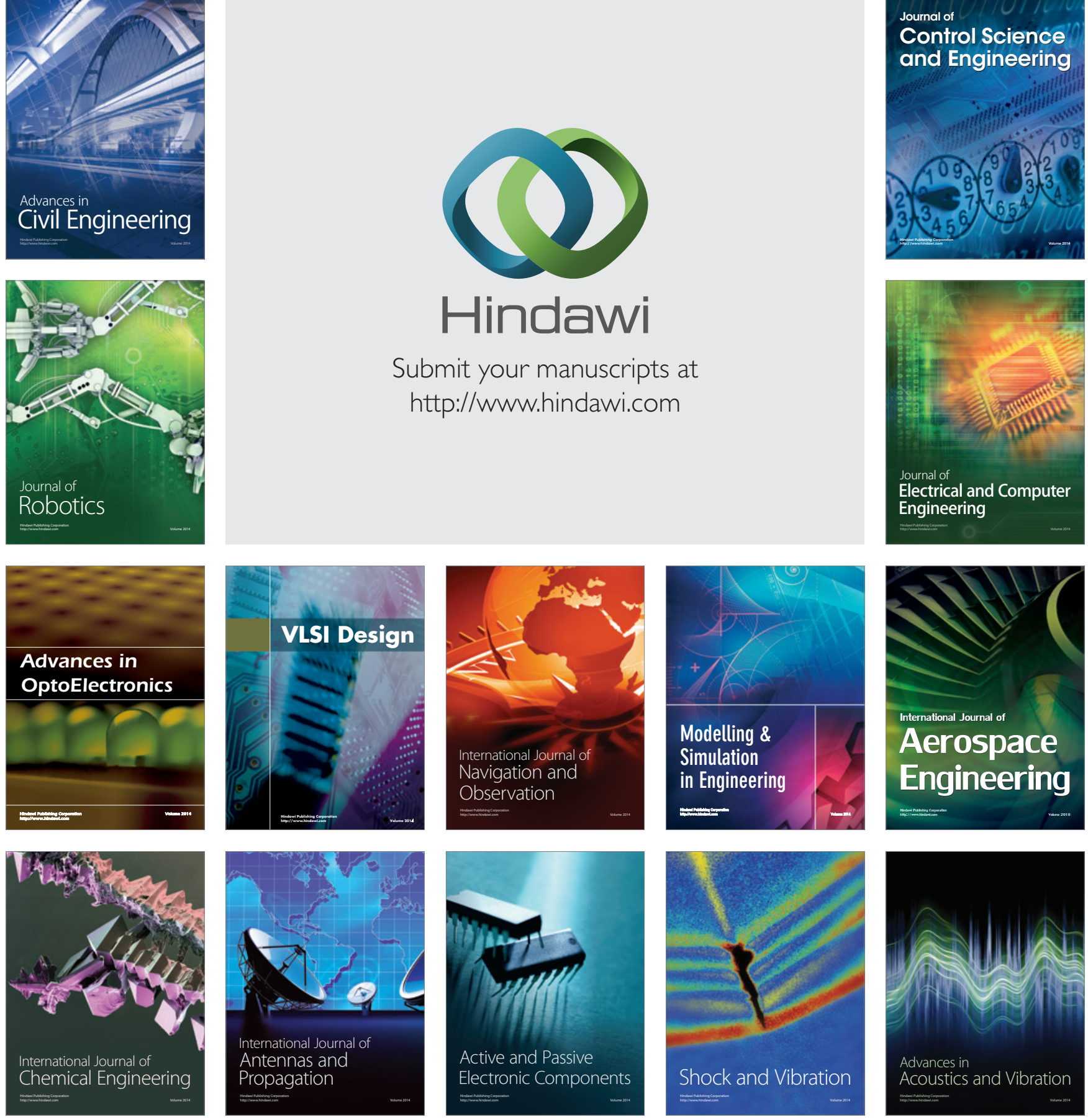